\title{
ADXS11-001 immunotherapy targeting HPV-E7: updated survival and safety data from a phase 2 study in Indian women with recurrent/refractory cervical cancer
}

\author{
Robert G Petit ${ }^{1 *}$, Partha Basu ${ }^{2}$ \\ From Society for Immunotherapy of Cancer 28th Annual Meeting \\ National Harbor, MD, USA. 8-10 November 2013
}

\begin{abstract}
ADXS11-001 immunotherapy is a live attenuated Listeria monocytogenes $(L m)$ bioengineered to secrete a HPV-16E7 fusion protein targeting HPV transformed cells. The Lm vector serves as its own adjuvant and infects antigen presenting cells (APC) where it cross presents, stimulating $\mathrm{MHC}$ class I and II pathways resulting in specific $\mathrm{T}$-cell immunity to tumors. Here we describe final 12 month overall survival data associated with ADXS11001 administration in Lm-LLO-E7-015, a randomized P2 study conducted in India in 110 patients with recurrent cervical cancer; previously treated with chemotherapy, radiotherapy or both. Patients were randomized to either 1 cycle (3 doses) of ADXS11-001 at $1 \times 10^{9} \mathrm{cfu}$ or 4 doses of ADXS11-001 at $1 \times 10^{9}$ cfu with cisplatin chemotherapy. Naprosyn and oral promethazine were given as premedications and a course of ampicillin was given 72 hours after infusion. Patients received CT scans at baseline and 3, 6, 9, 12 and 18 months. The primary endpoint is overall survival. As of May 17, 2013, the trial has completed enrollment and 110 patients received 264 doses of ADXS11-001. The percentage of patients at 12 months is $36 \%(39 / 110)$ and currently the 18 month survival is $22 \%(16 / 73)$. The response rate was $11 \%$ (6 CRs and 6 PR/110) with tumor responses observed in both treatment arms. 33 additional patients had stable disease $>3$ months, for a disease control rate of $41 \%$ (45/110). Activity was observed against all high risk HPV strains detected. Two Grade 3 serious adverse events and 104 mild-moderate adverse events possibly related/ related to ADXS11-001 treatment have been reported in
\end{abstract}

41\% (45/110) of patients. The non-serious adverse events consisted predominately of transient, non-cumulative flulike symptoms associated with infusion that either selfresolved or responded to symptomatic treatment. ADXS11-001 can be safely administered to patients with advanced cancer alone and in combination with chemotherapy. ADXS11-001 is well tolerated and presents a predictable and manageable safety profile. The addition of cisplatin to ADXS11-001 in this study did not significantly improve tumor responses or overall survival. Objective tumor responses included CR's and apparent prolonged survival with minimal adverse experiences. Average duration of response in both treatment groups was 10.5 months. The $36 \% 12$ month survival and $11 \%$ response rate observed in this recurrent disease setting is encouraging and suggests that ADXS11-001 is an active agent in recurrent cervical cancer. Final 18 month overall survival will be presented at the meeting.

\section{Authors' details}

'Advaxis, Inc., Princeton, NJ, USA. ${ }^{2}$ Chittaranjan National Cancer Institute, Kolkata, India.

Published: 7 November 2013

\section{doi:10.1186/2051-1426-1-S1-P231}

Cite this article as: Petit and Basu: ADXS11-001 immunotherapy targeting HPV-E7: updated survival and safety data from a phase 2 study in Indian women with recurrent/refractory cervical cancer. Journal for ImmunoTherapy of Cancer 2013 1(Suppl 1):P231.

${ }^{1}$ Advaxis, Inc., Princeton, NJ, USA

Full list of author information is available at the end of the article

(0) 2013 Petit and Basu; licensee BioMed Central Ltd. This is an Open Access article distributed under the terms of the Creative 